\title{
Derivation of Boussinesq's shoaling law using a coupled BBM system
}

\author{
H. Kalisch and A. Senthilkumar \\ Department of Mathematics, University of Bergen, Postbox 7800, 5020 Bergen, Norway
}

Correspondence to: H. Kalisch (henrik.kalisch@math.uib.no) and A. Senthilkumar (amutha.senthilkumar@math.uib.no)

Received: 30 November 2012 - Revised: 17 February 2013 - Accepted: 20 February 2013 - Published: 14 March 2013

\begin{abstract}
This paper is focused on finding rules for waveheight change in a solitary wave as it runs up a slowly increasing bottom. A coupled BBM system is used to describe the solitary waves. Expressions for energy density and energy flux associated with the BBM system are derived, and the principle of energy conservation is used to develop an equation relating the waveheight and undisturbed depth to the initial undisturbed depth and the incident waveheight. In the limit of zero waveheight, Boussinesq's shoaling law is recovered.
\end{abstract}

\section{Introduction}

The study of surface gravity waves is one of the classical problems in fluid mechanics and is of fundamental importance in coastal engineering. One particular case of interest, both theoretically and in practice, is the development of ocean waves as they propagate shorewards and experience a decrease in the water depth. A significant amount of work has been focused on this phenomenon, which is known as wave shoaling. The literature on the subject includes experimental studies in controlled environments in particular, such as wave flumes, and a large number of theoretical studies.

Among the first to study the problem was Boussinesq, who treated wave shoaling in the framework of his theory of weakly nonlinear long waves. Based on the assumption that the energy content of the wave is unchanged as it propagates, Boussinesq developed a simple rule for the waveheight change of a long wave as it runs up on a bottom slope. Suppose the initial undisturbed depth of the fluid is $h_{0}$, and the initial waveheight is denoted by $H_{0}$. If the local depth is denoted by $h$, and the associated waveheight is denoted by $H$, then Boussinesq's law can be written as
$H / H_{0}=h_{0} / h$. This law applies in the context of the socalled Boussinesq scaling, where the waveheight is small and the wavelength is long when compared to the initial undisturbed depth $h_{0}$. In the case where the effects on nonlinear steepening and of linear dispersion are approximately balanced, solitary waves can be found, and it appears that Boussinesq's law applies primarily to the shoaling of solitary waves. As explained in Miles (1980), Boussinesq's law has been rediscovered a number of times with varying degrees of rigor. Probably the most careful derivation was given in Grimshaw (1970, 1971), where Boussinesq's result actually follows from a more general analysis of the wave action principle. Indeed, Grimshaw (1971) studied the evolution of solitary waves in water of variable depth and he observed that for small values of initial waveheight, the shoaling rates are not exactly given by Boussinesq's law, but that they approach Boussinesq's law in the limit $H_{0} \rightarrow 0$.

In the present paper, the Boussinesq law is derived using a different approach, which is based directly on Boussinesq's original assumption that energy is conserved as the wave shoals. Using this assumption, and a careful analysis of the energy density associated to a particular Boussinesq system featuring exact solitary-wave solutions, waveheight changes can be computed in a straightforward manner. The system used here is known as the coupled BBM system. The method used to find the associated energy density is an extension of the recent work Ali and Kalisch (2012) where approximations of energy density and flux in the context of the Boussinesq scaling were found. The computations actually show that for waves of finite waveheight, the shoaling rate is somewhat lower than Boussinesq's law suggests. However, in the limit of zero waveheight, Boussinesq's law is recovered. In this sense, the results are in line with the findings of Grimshaw (1971). 
It should be noted that we do not incorporate an uneven bottom profile into the equations, but rather consider the transition of the wave on the slope as a gradual adjustment process of which only the initial and final states are considered. Since the effects of the bottom slope are only modeled indirectly, our analysis of wave shoaling using the conservation of energy depends strongly on the adiabatic approximation. In clear terms, it must be assumed that the wavelength $l$ of the wave running up the slope is much smaller than the characteristic length $h \Delta x /\left(h_{0}-h\right)$ of the depth variation. If this is the case, then the wave undergoes an adiabatic change, and the relation between wavelength and wave amplitude stays approximately intact. If the bottom gradient is too large, then the wave will change character and violate the Boussinesq scaling as it runs up the slope. Moreover, larger slopes will lead to reflected waves and significant steepening and asymmetry of the main wave, and none of these figure into the present analysis.

Let us briefly introduce the model system to be used here. Assuming $a$ is a typical amplitude and $l$ is a typical wavelength of the waves to be described, the parameter $\alpha=a / h_{0}$ represents the waveheight to depth ratio, and the parameter $\beta=h_{0}^{2} / l^{2}$ represents a water depth to wavelength ratio. The Boussinesq scaling consists of the assumptions that nonlinearity is weak $(\alpha \ll 1)$, and dispersive effects are also weak $(\beta \ll 1)$, and the two parameters of the same order. In other words, the Stokes number $S=\alpha / \beta$ should be $\mathcal{O}(1)$. In Bona et al. (2002), a general family of Boussinesq system was found, and one particular case is the coupled BBM system to be used in the present study. In the non-dimensional variables to be defined in Section 2, the system takes the form

$$
\begin{aligned}
\tilde{\eta}_{\tilde{t}}+\tilde{w}_{\tilde{x}}+\alpha(\tilde{\eta} \tilde{w})_{\tilde{x}}-\frac{1}{2}\left(\theta^{2}-\frac{1}{3}\right) \beta \tilde{\eta}_{\tilde{x} \tilde{x} \tilde{t}}=\mathcal{O}\left(\alpha \beta, \beta^{2}\right), \\
\tilde{w}_{\tilde{t}}+\tilde{\eta}_{\tilde{x}}+\alpha \tilde{w} \tilde{w}_{\tilde{x}}-\frac{1}{2}\left(1-\theta^{2}\right) \beta \tilde{w}_{\tilde{x} \tilde{x} \tilde{t}}=\mathcal{O}\left(\alpha \beta, \beta^{2}\right) .
\end{aligned}
$$

Here $\tilde{\eta}$ represents the non-dimensional excursion of the free surface, and $\tilde{w}$ represents the non-dimensional horizontal velocity at a non-dimensional height $0 \leq \theta \leq 1$ in the fluid column. Disregarding terms of order $\mathcal{O}\left(\alpha^{2}, \alpha \beta, \beta^{2}\right)$ yields the evolution system governing the approximate dynamics of the flow. The point of view adopted in the present paper is that Eq. (1) represents an approximate mass conservation equation, and Eq. (2) represents approximate momentum conservation. Since energy conservation is not an independent principle in homogeneous fluids, it should be possible to express energy conservation in terms of the unknowns $\tilde{\eta}$ and $\tilde{w}$ of Eqs. (1) and (2). The approximate energy balance equation can be written in the form

$$
\frac{\partial}{\partial \tilde{t}} \tilde{E}(\tilde{\eta}, \tilde{w})+\frac{\partial}{\partial \tilde{x}} \tilde{q}_{E}(\tilde{\eta}, \tilde{w})=\mathcal{O}\left(\alpha^{2}, \alpha \beta, \beta^{2}\right),
$$

and the principal task is to find appropriate expressions for the energy density $\tilde{E}(\tilde{\eta}, \tilde{w})$ and energy flux $\tilde{q}_{E}(\tilde{\eta}, \tilde{w})$. These quantities will be computed in Sect. 2. Section 3 is devoted to the derivation of an approximate shoaling law, and Sect. 4 contains a brief discussion.

\section{Energy balance}

For an inviscid, incompressible fluid, the surface water-wave problem is given by the Euler equations with no-flow conditions at the bottom and kinematic and dynamic boundary conditions at the free surface. Let the spatial coordinates be $(x, z)$ and the $x$-axis be oriented in the horizontal direction. Assume that the motion is uniform in the direction perpendicular to the $x z$-plane (long-crested waves). The gravitational acceleration $g$ acts in the negative $z$-direction. Let $\eta(x, t)$ denote the surface elevation, and let $\phi(x, z, t)$ be the velocity potential.

From the incompressibility of the fluid, the potential $\phi$ satisfies Laplace's equation in the domain $\left\{(x, z) \in \mathbb{R}^{2} \mid-h_{0}<\right.$ $z<\eta(x, t)\}$. The complete problem is written as follows.

$\Delta \phi=0$ in $-h_{0}<z<\eta(x, t)$,

$\phi_{z}=0$ on $z=-h_{0}$,

$$
\left.\begin{array}{c}
\eta_{t}+\phi_{x} \eta_{x}-\phi_{z}=0, \\
\phi_{t}+\frac{1}{2}\left(\phi_{x}^{2}+\phi_{y}^{2}\right)+g \eta=0,
\end{array}\right\} \quad \text { on } z=\eta(x, t) .
$$

As the derivation of the coupled BBM-system is well known (see Bona et al., 2002 and Whitham, 1974), we only sketch the outline for the interested reader. Denote the limiting longwave speed by $c_{0}=\sqrt{g h_{0}}$, and define non-dimensional variables by

$\tilde{x}=\frac{x}{l}, \quad \tilde{z}=\frac{z+h_{0}}{h_{0}}, \quad \tilde{\eta}=\frac{\eta}{a}, \quad \tilde{t}=\frac{c_{0} t}{l}, \quad \tilde{\phi}=\frac{c_{0} \phi}{g a l}$.

As explained by Whitham (1974), one may use the ansatz

$\tilde{\phi}=\sum_{m=0}^{\infty} \tilde{f}_{m}(\tilde{x}, \tilde{t}) \tilde{z}^{m} \beta^{m}$

to represent the non-dimensional velocity potential in terms of the unknown functions $\tilde{f}_{m}(\tilde{x}, \tilde{t})$. Now Laplace's equation and the bottom boundary condition Eq. (5) may be used to arrive at the expression

$\tilde{\phi}=\tilde{f}-\frac{\tilde{z}^{2}}{2} \tilde{f}_{\tilde{x} \tilde{x}} \beta+\mathcal{O}\left(\beta^{2}\right)$,

where $\tilde{f}=\tilde{f}_{0}$. From Eq. (8) and the second boundary condition at the free surface, we have the relation

$\tilde{\eta}+\tilde{f}_{\tilde{t}}-\frac{\beta}{2} \tilde{f}_{\tilde{x} \tilde{x} \tilde{t}}+\frac{\alpha}{2} \tilde{f}_{\tilde{x}}^{2}=\mathcal{O}\left(\alpha \beta, \beta^{2}\right)$.

Differentiating Eq. (9) with respect to $\tilde{x}$, and inserting the expansion for $\phi$ in the first boundary condition at the free 
surface, Eq. (6) yields a system of equations in terms of the horizontal velocity at the bottom $\tilde{v}=\tilde{f}_{\tilde{x}}$. However, for the purposes of the present article, a different but equivalent system will have to be used. Denoting by $\tilde{w}$ the non-dimensional horizontal velocity at a non-dimensional height $0 \leq \theta \leq 1$ in the fluid column, elementary considerations (see Bona et al., 2002) show that $\tilde{v}$ and $\tilde{w}$ are related by

$\tilde{v}=\tilde{w}+\frac{\beta}{2} \theta^{2} \tilde{w}_{\tilde{x} \tilde{x}}+\mathcal{O}\left(\beta^{3}\right)$.

One may use Eq. (10) and the first-order relations $\tilde{v}_{\tilde{t}}+\tilde{\eta}_{\tilde{x}}=$ $\mathcal{O}(\alpha, \beta), \tilde{\eta}_{\tilde{t}}+\tilde{v}_{\tilde{x}}=\mathcal{O}(\alpha, \beta)$ to arrive at a general model system for small-amplitude long waves. The system is given in non-dimensional variables by

$\tilde{\eta}_{\tilde{t}}+\tilde{w}_{\tilde{x}}+\alpha(\tilde{\eta} \tilde{w})_{\tilde{x}}+\frac{1}{2}\left(\theta^{2}-\frac{1}{3}\right) \lambda \beta \tilde{w}_{\tilde{x} \tilde{x} \tilde{x}}$

$-\frac{1}{2}\left(\theta^{2}-\frac{1}{3}\right)(1-\lambda) \beta \tilde{\eta}_{\tilde{x} \tilde{x} \tilde{t}}=\mathcal{O}\left(\alpha \beta, \beta^{2}\right)$,

$\tilde{w}_{\tilde{t}}+\tilde{\eta}_{\tilde{x}}+\alpha \tilde{w} \tilde{w}_{\tilde{x}}+\frac{1}{2}\left(1-\theta^{2}\right) \mu \beta \tilde{\eta}_{\tilde{x} \tilde{x} \tilde{x}}$

$-\frac{1}{2}\left(1-\theta^{2}\right)(1-\mu) \beta \tilde{w}_{\tilde{x} \tilde{x} \tilde{x}}=\mathcal{O}\left(\alpha \beta, \beta^{2}\right)$.

Here $\lambda$ and $\mu$ are modeling parameters which have no physical meaning, but can be chosen arbitrarily in the range $0 \leq \lambda, \mu \leq 1$. Choosing in particular $\lambda=0$ and $\mu=0$ yields the coupled BBM system Eqs. (1) and (2).

In order to derive the associated energy balance equation, we need expressions for the velocity field and pressure. The velocity field is easily seen to be given by

$\tilde{\phi}_{\tilde{x}}=\tilde{w}+\frac{\beta}{2}\left(\theta^{2}-\tilde{z}^{2}\right) \tilde{w}_{\tilde{x} \tilde{x}}+\mathcal{O}\left(\beta^{2}\right)$,

$\tilde{\phi}_{\tilde{z}}=-\tilde{z} \tilde{w}_{\tilde{x}} \beta+\mathcal{O}\left(\beta^{2}\right)$.

The dynamic pressure $P^{\prime}$, which measures the deviation from hydrostatic pressure, is given quite generally by

$P^{\prime}=P-P_{\mathrm{atm}}+\rho g z=-\rho \phi_{t}-\frac{\rho}{2}|\nabla \phi|^{2}$.

We use the scaling $\rho g a \tilde{P}^{\prime}=P^{\prime}$. Then as shown by Ali and Kalisch (2012), the dynamic pressure can be found with the help of Eq. (9) to be

$\tilde{P}^{\prime}=\tilde{\eta}+\frac{1}{2} \beta\left(\tilde{z}^{2}-1\right) \tilde{w}_{\tilde{x} \tilde{t}}+\mathcal{O}\left(\alpha \beta, \beta^{2}\right)$.

Next, we examine energy balance of the BBM system. If we assume that the potential energy of a particle is zero at the undisturbed free surface, and the potential energy is zero when no wave motion is present, then the total energy inside a control volume of unit width, delimited by the interval $\left[x_{1}, x_{2}\right]$ on the lateral sides, and by the bottom and the free surface can be written as

$E=\frac{1}{2} \int_{x_{1}}^{x_{2}} \int_{-h_{0}}^{\eta} \rho|\nabla \phi|^{2} \mathrm{~d} z \mathrm{~d} x+\int_{x_{1}}^{x_{2}} \int_{0}^{\eta} \rho g z \mathrm{~d} z \mathrm{~d} x$, where the first term represents the kinetic energy, and the second term represents potential energy. The conservation of total mechanical energy (see Stoker, 1957, chap.1.) is written as

$$
\begin{aligned}
& \frac{\mathrm{d}}{\mathrm{d} t} \frac{1}{2} \int_{x_{1}}^{x_{2}} \int_{-h_{0}}^{\eta} \rho|\nabla \phi|^{2} \mathrm{~d} z \mathrm{~d} x+\frac{\mathrm{d}}{d t} \int_{x_{1}}^{x_{2}} \int_{0}^{\eta} \rho g z \mathrm{~d} z \mathrm{~d} x \\
& =\left[\int_{-h_{0}}^{\eta}\left\{\frac{\rho}{2}|\nabla \phi|^{2}+\rho g z+P\right\} \phi_{x} \mathrm{~d} z\right]_{x_{1}}^{x_{2}} .
\end{aligned}
$$

Expressing the above relation in non-dimensional variables gives

$$
\begin{aligned}
& \frac{\mathrm{d}}{\mathrm{d} \tilde{t}} \int_{x_{1} / l}^{x_{2} / l} \int_{0}^{l+\alpha \tilde{\eta}}\left\{\frac{\alpha^{2}}{2}\left(\tilde{\phi}_{\tilde{x}}^{2}+\frac{1}{\beta} \tilde{\phi}_{\tilde{z}}^{2}\right)\right\} \mathrm{d} \tilde{z} \mathrm{~d} \tilde{x} \\
& +\frac{\mathrm{d}}{\mathrm{d} \tilde{t}} \int_{x_{1} / l}^{x_{2} / l} \int_{1}^{l+\alpha \tilde{\eta}}(\tilde{z}-1) \mathrm{d} \tilde{z} \mathrm{~d} \tilde{x}=\left[\int _ { 0 } ^ { 1 + \alpha \tilde { \eta } } \left\{\frac{\alpha^{3}}{2}\left(\tilde{\phi}_{\tilde{x}}^{3}+\frac{1}{\beta} \tilde{\phi}_{\tilde{z}}^{2} \tilde{\phi}_{\tilde{x}}\right)\right.\right. \\
& \left.+\alpha(\tilde{z}-1) \tilde{\phi}_{\tilde{x}}+\alpha^{2} \tilde{P}^{\prime} \tilde{\phi}_{\tilde{x}}+\alpha(1-\tilde{z}) \tilde{\phi}_{\tilde{x}}\right\} \mathrm{d} \tilde{z}_{x_{1} / l}^{x_{2} / l} .
\end{aligned}
$$

If we substitute the expressions for $\tilde{\phi}_{\tilde{x}}, \tilde{\phi}_{\tilde{z}}$ and $\tilde{P}^{\prime}$ found in Eqs. (13) and (15) respectively, and integrate with respect to $\tilde{z}$, then we obtain

$$
\begin{aligned}
& \frac{\mathrm{d}}{\mathrm{d} \tilde{t}} \int_{x_{1} / l}^{x_{2} / l}\left\{\frac{\alpha^{2}\left(\tilde{w}^{2}+\tilde{\eta}^{2}\right)}{2}+\frac{\alpha^{2} \beta}{2}\left(\theta^{2}-\frac{1}{3}\right) \tilde{w} \tilde{w}_{\tilde{x} \tilde{x}}\right. \\
& \left.+\frac{\alpha^{2} \beta}{6}\left(\tilde{w}_{\tilde{x}}\right)^{2}+\frac{\alpha^{3} \tilde{w}}{2} \tilde{\eta}\right\} \mathrm{d} \tilde{x}=\left[\frac{\alpha^{3}}{2} \tilde{w}^{3}+\alpha^{2} \tilde{\eta} \tilde{w}+\alpha^{3} \tilde{\eta}^{2} \tilde{w}\right. \\
& \left.+\frac{\alpha^{2}}{2} \beta\left(\theta^{2}-\frac{1}{3}\right) \tilde{\eta} \tilde{w}_{\tilde{x} \tilde{x}}-\frac{\alpha^{2}}{3} \beta \tilde{w} \tilde{w}_{\tilde{x} \tilde{t}}\right]_{x_{1} / l}^{x_{2} / l}+\mathcal{O}\left(\alpha^{2} \beta^{2}, \alpha^{3} \beta, \alpha^{4}\right) .
\end{aligned}
$$

Taking the limit as $x_{2} \rightarrow x_{1}$, and omitting the common factor $\alpha^{2}$, we obtain the differential energy balance equation

$$
\begin{array}{r}
\frac{\mathrm{d}}{\mathrm{d} \tilde{t}}\left[\frac{\left(\tilde{w}^{2}+\tilde{\eta}^{2}\right)}{2}+\frac{\beta}{2}\left(\theta^{2}-\frac{1}{3}\right) \tilde{w} \tilde{w}_{\tilde{x} \tilde{x}}+\frac{\beta}{6}\left(\tilde{w}_{\tilde{x}}\right)^{2}+\frac{\alpha \tilde{w}^{2}}{2} \tilde{\eta}\right]+ \\
\frac{\mathrm{d}}{\mathrm{d} \tilde{x}}\left[\frac{\alpha}{2} \tilde{w}^{3}+\tilde{\eta} \tilde{w}+\alpha \tilde{\eta}^{2} \tilde{w}+\frac{1}{2} \beta\left(\theta^{2}-\frac{1}{3}\right) \tilde{\eta} \tilde{w}_{\tilde{x} \tilde{x}}-\frac{1}{3} \beta \tilde{w} \tilde{w}_{\tilde{x} \tilde{t}}\right] \\
=\mathcal{O}\left(\beta^{2}, \alpha \beta, \alpha^{2}\right) .
\end{array}
$$

From Eq. (16), we get the non-dimensional energy density

$$
\begin{aligned}
& \tilde{E}=\frac{\alpha^{2}\left(\tilde{w}^{2}+\tilde{\eta}^{2}\right)}{2}+\frac{\alpha^{2} \beta}{2}\left(\theta^{2}-\frac{1}{3}\right) \tilde{w} \tilde{w}_{\tilde{x} \tilde{x}} \\
& +\frac{\alpha^{2} \beta}{6}\left(\tilde{w}_{\tilde{x}}\right)^{2}+\frac{\alpha^{3} \tilde{w}^{2}}{2} \tilde{\eta},
\end{aligned}
$$


and the non-dimensional energy flux

$\tilde{q}_{\tilde{E}}=\frac{\alpha^{3}}{2} \tilde{w}^{3}+\alpha^{2} \tilde{\eta} \tilde{w}+\alpha^{3} \tilde{\eta}^{2} \tilde{w}$

$+\frac{\alpha^{2}}{2} \beta\left(\theta^{2}-\frac{1}{3}\right) \tilde{\eta} \tilde{w}_{\tilde{x} \tilde{x}}-\frac{\alpha^{2}}{3} \beta \tilde{w} \tilde{w}_{\tilde{x} \tilde{t}}$.

The dimensional versions of these quantities are given by

$E=\frac{\rho}{2} h_{0} w^{2}+\frac{\rho}{2} g \eta^{2}+\frac{\rho}{2} h_{0}^{3}\left(\theta^{2}-\frac{1}{3}\right) w w_{x x}$

$+\frac{\rho}{6} h_{0}^{3} w_{x}^{2}+\frac{\rho}{2} w^{2} \eta$

and

$$
\begin{gathered}
q_{E}=\frac{\rho}{2} h_{0} w^{3}+\rho c_{0}^{2} \eta w+\frac{1}{h_{0}} \rho c_{0}^{2} w \eta^{2} \\
+\frac{\rho}{2} c_{0}^{2} h_{0}^{2}\left(\theta^{2}-\frac{1}{3}\right) \eta w_{x x}-\frac{\rho}{3} h_{0}^{3} w w_{x t} .
\end{gathered}
$$

We would like to point out that it would be interesting to derive similar quantities for the system derived by Green and Naghdi (1976), which does not have the same limitations on the wave amplitude as the BBM system used in the present analysis. The Green-Naghdi system is also applicable for variable bottom topography, and has been used in the modeling of tsunamis by Zheleznyak and Pelinovsky (1985). In fact, a pressure formula similar to Eq. (15), but valid for the Green-Naghdi system has already been found by Pelinovsky and Choi (1993).

\section{Solitary wave shoaling}

One of the most readily investigated changes in a wave transmitting into shallower water is the variation in waveheight, and this is the main object of this section. We focus on the case of a solitary wave which runs up on a gentle slope with no variation in the transverse direction. Even in this twodimensional case, there are a number of important physical effects which are neglected in the model at hand. In particular, we do not take account of viscosity and rotational effects; and as explained in the introduction, we also assume that wave reflection can be neglected to a first approximation, such as for instance in the case of a very gentle slope. Moreover, we consider long-crested waves shoaling on a plane beach, so that wave refraction does not play a role. As the solitary wave starts to propagate over a sloping bottom, it will become slightly skewed, and the waveheight will increase. Eventually, the wave will steepen and break. The different stages of this shoaling process have been minutely detailed by Synolakis and Skjelbreia (1993).

As shown by Chen (1998), the coupled BBM system Eqs. (1) and (2) features solitary-wave solutions in a closed form in the case that $\theta^{2}=\frac{7}{9}$. Since the analysis of the energy balance in a shoaling wave given here relies on the exact formula for the solitary wave, the coupled BBM system with $\theta^{2}=\frac{7}{9}$ is used in the present work. In dimensional variables, this system takes the form

$$
\begin{aligned}
\eta_{t}+h_{0} w_{x}+(\eta w)_{x}-\frac{h_{0}^{2}}{2} \frac{4}{9} \eta_{x x t} & =0, \\
w_{t}+g \eta_{x}+w w_{x}-\frac{h_{0}^{2}}{2} \frac{2}{9} w_{x x t} & =0 .
\end{aligned}
$$

The solitary wave solutions of Eqs. (18) and (19) are given by

$$
\begin{aligned}
& \eta_{0}(x, t)=H_{0} \operatorname{sech}^{2}\left(\kappa_{0}\left(x-C_{0} t\right)\right), \\
& w_{0}(x, t)=W_{0} \operatorname{sech}^{2}\left(\kappa_{0}\left(x-C_{0} t\right)\right) .
\end{aligned}
$$

The constant $H_{0}$ is the initial waveheight, and the constants $W_{0}, C_{0}$ and $\kappa_{0}$ are given by

$$
\begin{aligned}
& W_{0}=\sqrt{\frac{3 g}{H_{0}+3 h_{0}}} H_{0}, \\
& C_{0}=\frac{3 h_{0}+2 H_{0}}{\sqrt{3 h_{0}\left(H_{0}+3 h_{0}\right)}} \sqrt{g h_{0}},
\end{aligned}
$$

and

$$
\kappa_{0}=\frac{3}{2 h_{0}} \sqrt{\frac{H_{0}}{2 H_{0}+3 h_{0}}} .
$$

Now let us consider a channel of depth $h_{0}$ and suppose the depth of the water is slowly decreasing to a smaller value $h$. We suppose that the waveheight of the incident wave is $H_{0}$, and denote waveheight of the wave at the new depth by $H$. In an experimental setting, the waveheight is usually continuously varying, and the waveheight change is recorded over the extent of the slope (cf. Fig. 1). In order to invoke energy conservation in a theoretical sense, we assume that the wave travels up a gentle slope, and reorganizes into a solitary wave on the new even bottom with undisturbed depth $h$.

Using the expression for $E$ obtained in the previous section, and evaluating the integral $\mathcal{E}=\int E \mathrm{~d} x$ for the total energy at two different instants in time, one at which the wave is located over the initial depth $h_{0}$, and one at which the wave is located above a different depth $h$ then yields an equation relating the waveheights $H_{0}$ and $H$ at the two different depths.

Since the solitary wave features exponential decay, energy conservation may be stated in the form

$$
\int_{-\infty}^{\infty} E\left(\eta_{0}, w_{0}\right) \mathrm{d} x=\int_{-\infty}^{\infty} E(\eta, w) \mathrm{d} x,
$$

where $\eta$ and $w$ are given by Eq. (20) with the constants $W$, $C$ and $\kappa$ given in terms if $h$ and $H$ instead of $h_{0}$ and $H_{0}$. After performing the integration with respect to $x$, we find the relation

$$
\begin{aligned}
& \frac{h_{0}}{2} \frac{W_{0}^{2}}{\kappa_{0}}-\frac{2}{45} h_{0}^{3} \kappa_{0} W_{0}^{2}+\frac{2}{5} W_{0}^{2} H_{0} \frac{1}{\kappa_{0}}+\frac{1}{2} g H_{0}^{2} \frac{1}{\kappa_{0}}= \\
& \frac{h}{2} \frac{W^{2}}{\kappa}-\frac{2}{45} h^{3} \kappa W^{2}+\frac{2}{5} W^{2} H \frac{1}{\kappa}+\frac{1}{2} g H^{2} \frac{1}{\kappa} .
\end{aligned}
$$




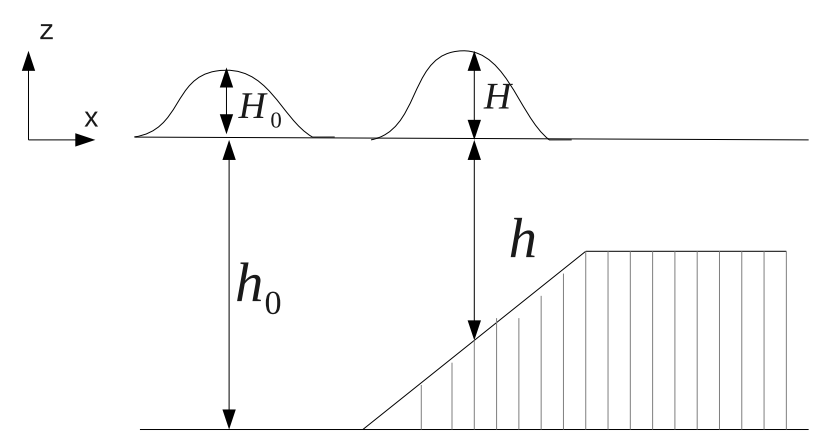

Fig. 1. The geometry of the problem. The waveheight $H$ is measured on the slope.

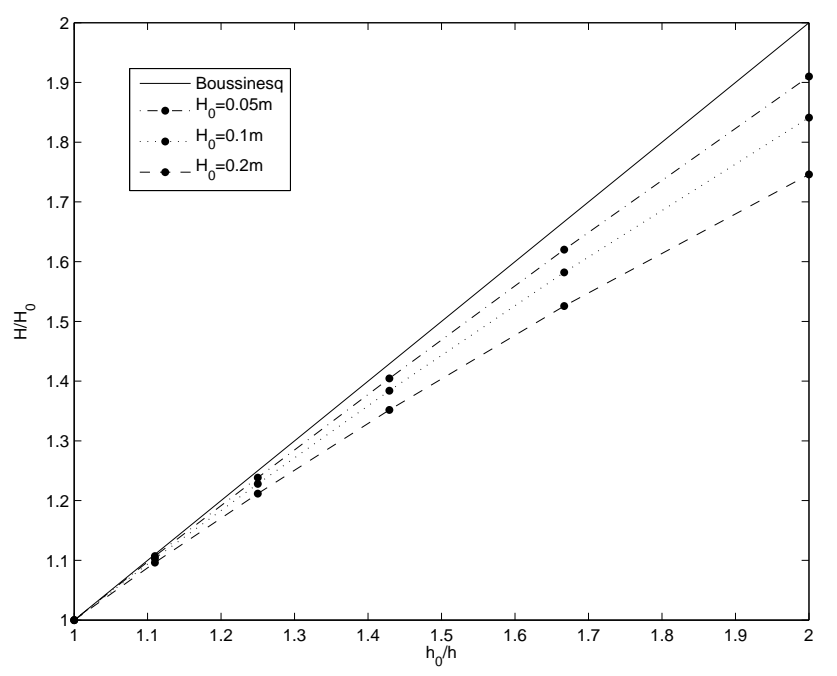

Fig. 2. Computations for the shoaling of solitary waves from relation Eq. (23). The solid line depicts the shoaling relation according to Boussinesq's law. Shoaling rates for waves of initial waveheight $H_{0}=0.05 \mathrm{~m}, 0.1 \mathrm{~m}$ and $0.2 \mathrm{~m}$ are computed. As the waveheight of the incident solitary waves decreases, the shoaling relation approaches Boussinesq's result.

From Eq. (23), it is plain that $H$ may be expressed in terms of $h_{0}, h$ and $H_{0}$, though in general the values of $H$ will have to be approximated numerically. We have computed wave shoaling for initial undisturbed depth $h_{0}=1 \mathrm{~m}$ and initial waveheights $H_{0}$ equal to $0.05 \mathrm{~m}, 0.1 \mathrm{~m}$ and $0.2 \mathrm{~m}$, and for a ratio of undisturbed to initial depth of up to 0.5 . The relative wave height $H / H_{0}$ computed for these waves is plotted in Fig. 2. Boussinesq's relation which gives shoaling rates $\propto h^{-1}$, is plotted as a solid line. It is apparent that the computed curves get close to the line $h^{-1}$ for decreasing initial waveheight.

\section{Discussion and conclusions}

Shoaling of solitary waves has been analyzed using conservation of energy in the Boussinesq scaling. It appears that for waves of very small waveheight, Boussinesq's shoaling law is approximately recovered. To explain the discrepancy observed for larger waveheights, we note that the Boussinesq shoaling law follows for small waveheights if only the last term in the expression Eq. (17) for the energy density is used to compute the energy of the solitary wave. This can also be done in the context of other Boussinesq-type systems found by Bona et al. (2002). In particular, in the case of the KdV equation, the total mechanical energy for a solitary wave is given by

$\mathcal{E}=\rho g \frac{8}{3^{3 / 2}} H^{3 / 2} h^{3 / 2}$,

which along with conservation of energy during an adiabatic shoaling process, yields Boussinesq's law $H / H_{0}=h_{0} / h$. In the limit of small amplitude, solitary-wave solutions of all the Boussinesq-type systems are equivalent to solitary waves of the KdV equation, so that the formula Eq. (24) is valid asymptotically for $H_{0} \rightarrow 0$ also for solitary waves governed by Eqs. (18) and (19). As explained by Bona et al. (2002), these Boussinesq-type systems are valid for waves for which the Stokes number $S=\alpha / \beta$ is of $\mathcal{O}(1)$. Shoaling rates for other waves can be quite different. In particular, for small amplitude waves, one often finds the so-called Green's law $H / H_{0} \sim 1 / h^{1 / 4}$, which can be derived from linear wave theory (see Lamb, 1932 and Synolakis, 1991).

For comparison, we have included some aspects of a shoaling study which was first presented by Pelinovsky and Talipova $(1977,1979)$. In these works, the waveheight-wave energy relation for numerical solutions of the full waterwave problem found by Longuet-Higgins (1974); LonguetHiggins and Fenton (1974) is used. The results are displayed in Fig. 3, and indicate shoaling rates similar to Boussinesq's law for most cases, since the slopes of the curves are seen to be close to 1 for the most part. Only the very first part of the curve for $H_{0}=0.05 \mathrm{~m}$ has a smaller slope, and might suggest shoaling rates closer to Green's law for very small amplitude waves and small differences in depth. Since Green's laws can be derived in the case when there is no particular relationship between the wavelength and the amplitude, it is not surprising that for solitary waves, which generally respect the relation $\alpha \sim \beta$, the Boussinesq law is a more generic outcome.

Besides the works Pelinovsky and Talipova $(1977,1979)$ already mentioned, there have also been other analytic studies. For instance, in Pelinovsky et al. (1993), nonlinear ray theory is used to derive a rather general shoaling law, including wave diffraction and dissipation. The shoaling relation derived by Pelinovsky et al. (1993) reduces to Green's law in the case that nonlinearity and dispersion are neglected. In the case of a periodic sequence of solitary waves, the relation 


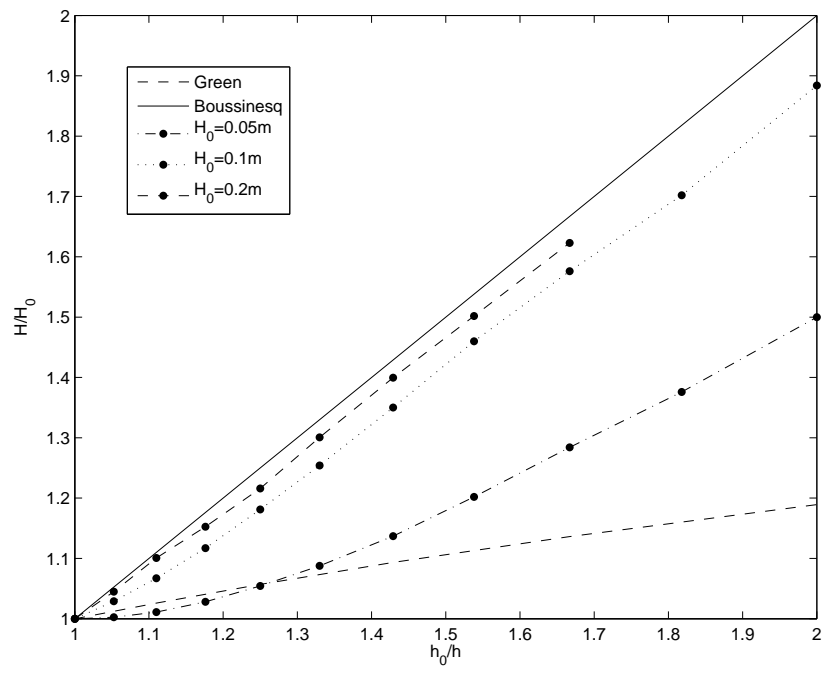

Fig. 3. Computations for the shoaling of solitary waves using the method of Pelinovsky and Talipova $(1977,1979)$, and using numerical data found by Longuet-Higgins (1974); Longuet-Higgins and Fenton (1974). The solid line depicts the shoaling relation according to Boussinesq's law. The lower dashed line depicts the shoaling relation according to Green's law. Shoaling rates for waves of initial waveheight $H_{0}=0.05 \mathrm{~m}, 0.1 \mathrm{~m}$ and $0.2 \mathrm{~m}$ are computed. The dashed curve terminates because the maximum wave energy is reached.

reduces to a "nonlinear" Green's law, such as found by Ostrovsky and Pelinovsky (1970). See also Ostrovsky and Pelinovsky (1975) for nonlinear wave refraction.

Comparisons with wave tank experiments indicate that reliable results can be obtained for variations of the Stokes number $S$ over nearly two orders of magnitude (see Bona et al. (1981)). However, these comparisons also indicate that the an appropriate damping mechanism should be included into the description. The effect of different models of bottom friction on the waveheight changes in surface waves were investigated by Caputo and Stepanyants (2003). It was found that of the three dissipation models considered, Chezy-type dissipation had the strongest damping effect in a channel of decreasing depth, while Reynolds dissipation had the weakest effect. These authors also considered the influence of a channel of changing width. In particular, a generalised Green's law is proposed which takes account of both changing depth and width.

There have also been several experimental and purely numerical studies directed towards understanding shoaling rates of long-crested waves in shallow water. Early experimental work of Ippen and Kulin (1954) and Camfield and Street (1969) suggested that wave shoaling may be approximated by Green's law (see Madsen and Mei (1969)), but some of these date feature high scatter, and some also suggest shoaling rates higher than Green's law. The systematic study of Synolakis and Skjelbreia (1993) found that solitary wave shoaling can be described well if the evolution is classified in different phases. There are two pre-breaking phases, the first is relatively well approximated by Green's law the second which features more rapid shoaling can be approximated by Boussinesq's law.

Using a numerical approximation of a Boussinesq model similar to the system studied in this paper, Peregrine (1967) found that the shoaling rates can be qualitatively predicted by Green's laws, but that there is no systematic variation of the waveheight change with either slope or initial waveheight. Shuto (1973) suggested that growth rates of both Boussinesq's result and Green's law are correct, and the validity of either depends on the parameter range of any particular situation. In fact, Shuto displays graphs which suggest that both the experiments by Camfield and Street (1969) and Ippen and Kulin (1954) could be interpreted using Boussinesq's law or Green's law, in certain areas. In his review article on solitary waves, Miles (1980) noted that Boussinesq's shoaling law should be appropriate for sufficiently small values of the bottom slope, but that Green's law is a better approximation for larger slopes or sufficiently small waveheight of the incident wave.

Acknowledgements. This research was supported by the Research Council of Norway. The authors would like to thank Efim Pelinovsky and Yury Stepanyants for helpful comments on this work.

Edited by: R. Grimshaw

Reviewed by: E. Pelinovsky and Y. A. Stepanyants

\section{References}

Ali, A. and Kalisch, H.: Mechanical balance laws for Boussinesq models of surface water waves, J. Nonlinear Sci., 22, 371-398, doi:10.1007/s00332-011-9121-2, 2012.

Benjamin, T. B., Bona, J. B. and Mahony, J. J.: Model equations for long waves in nonlinear dispersive systems, Philos. T. R. Soc. A, 272, 47-78, 1972.

Bjørkavåg, M. and Kalisch, H.: Wave breaking in Boussinesq models for undular bores, Phys. Lett. A, 375, 1570-1578, doi:10.1016/j.physleta.2011.02.060, 2011.

Bona, J. L. and Chen, M.: A Boussinesq system for two-way propagation of nonlinear dispersive waves, Physica D, 116, 191-224, doi:10.1016/S0167-2789(97)00249-2, 1998.

Bona, J. L., Chen, M., and Saut, J.-C.: Boussinesq equations and other systems for small-amplitude long waves in nonlinear dispersive media.I: Derivation and linear theory. J. Nonlinear Sci., 12, 283-318, doi:10.1007/s00332-002-0466-4, 2002.

Bona, J. L., Pritchard, W. G., and Scott, L. R.: An evaluation of a model equation for water waves, Philos. T. R. Soc. A, 302, 457510, 1981.

Camfield, F. E. and Street, R. L.: Shoaling of solitary waves on small slopes, J. Waterway. Harb. Div., Proc. ASCE 95, 1-22, 1969.

Caputo, J.-G. and Stepanyants, Y. A.: Bore formation, evolution and disintegration into solitons in shallow inhomogeneous channels, 
Nonlin. Processes Geophys., 10, 407-424, doi:10.5194/npg-10407-2003, 2003.

Chen, M.: Exact solutions of various Boussinesq systems, Appl. Math. Lett., 11, 45-49, doi:10.1016/S0893-9659(98)00078-0, 1998.

Choi, B. H., Pelinovsky, E., Kim, D. C., Didenkulova, I., and Woo, S.-B.: Two- and three-dimensional computation of solitary wave runup on non-plane beach, Nonlin. Processes Geophys., 15, 489502, doi:10.5194/npg-15-489-2008, 2008.

Dean, R. G. and Dalrymple, R. A.: Water Wave Mechanics for Engineers and Scientists, World Scientific, Singapore, 1991.

Green, A. E. and Naghdi, P. M.: A derivation of equations for wave propagation on water of variable depth, J. Fluid Mech., 78, 237246, doi:10.1017/S0022112076002425, 1976.

Grimshaw, R.: The solitary wave in water of variable depth, J. Fluid Mech., 42, 639-656, doi:10.1017/S0022112070001520, 1970.

Grimshaw, R.: The solitary wave in water of variable depth. Part 2, J. Fluid Mech., 46, 611-622, doi:10.1017/S0022112071000739, 1971.

Ippen, A. T. and Kulin, G.: The shoaling and breaking of the solitary wave, Proc. 5th Conf. on Coast. Eng., Grenoble, 27-47, 1954.

Kishi, T. and Saeki, H.: The shoaling, breaking and runup of the solitary wave on impermeable rough slopes. Proc. 10th Conf. Coastal Engineering, Tokyo, 322-348, 1966.

Lamb, H.: Hydrodynamics, Cambridge Univ. Press, California, 1932.

Longuet-Higgins, M. S.: On the mass, momentum, energy and circulation of a solitary wave. I, P. Roy. Soc. Lond. A Mat., 337, 1-13, doi:10.1098/rspa.1974.0035, 1974.

Longuet-Higgins, M. S. and Fenton, J. D.: On the mass, momentum, energy and circulation of a solitary wave. II, P. Roy. Soc. Lond. A Mat., 340, 471-493, doi:10.1098/rspa.1974.0166, 1974.

Madsen, O. S. and Mei, C. C.: The transformation of a solitary wave over an uneven bottom, J. Fluid Mech., 39, 781-791, doi:10.1017/S0022112069002461, 1969.

Miles, J. W.: Solitary waves, Annu. Rev. Fluid Mech., 12, 11-43, doi:10.1146/annurev.fl.12.010180.000303, 1980.
Ostrovsky, L. A. and Pelinovsky, E. N.: Wave transformation on the surface of a fluid of variable depth, Atmos. Oceanic Phys., 6, $552-555,1970$.

Ostrovsky, L. A. and Pelinovsky, E. N.: Refraction of nonlinear ocean waves in a beach zone, Atmos. Oceanic Phys., 11, 37-41, 1975.

Peregrine, D. H.: Long waves on a beach, J. Fluid Mech., 27, 815827, doi:10.1017/S0022112067002605, 1967.

Pelinovsky, E. N.: Nonlinear Dynamics of Tsunami Waves (Applied Physics Institute Press, Gorky, 1982).

Pelinovsky, E. N. and Choi, H. S.: A Mathematical model for nonlinear waves due to moving disturbances in a basin of variable depth, J. Korean Soc. Coastal Ocean Eng., 5, 191-197, 1993.

Pelinovsky, E. N., Stepanyants, Y. A. and Talipova, T. G.: Nonlinear dispersion model of sea waves in the coastal zone, J. Korean Soc. Coastal Ocean Eng., 5, 307-317, 1993.

Pelinovsky, E. N. and Talipova, T. G.: Height variations of largeamplitude solitary waves in the near-shore zone, Oceanology, 17, 1-3, 1977.

Pelinovsky, E. N. and Talipova, T. G.: Change of height of the solitary wave of large amplitude in the beach zone, Mar. Geodesy, 2, 313-321, 1979.

Shuto, N.: Shoaling and deformation of non-linear waves, Coast. Eng. Japan, 14, 25-42, 1973.

Stoker, J. J.: Water waves: The mathematical theory with applications, Pure and Applied Mathematics, IV, Interscience Publishers, New York, 1957.

Synolakis, C. E.: Green's law and the evolution of solitary waves, Phys. Fluids A, 3, 490-491, 1991.

Synolakis, C. E. and Skjelbreia, J. E.: Evolution of maximum amplitude of solitary waves on plane beaches, J. Waterw. Port. CAsce., 119, 323-342, 1993.

Whitham, G. B.: Linear and nonlinear waves, Wiley, New York, 1974.

Zheleznyak, M. I. and Pelinovsky, E. N.: Physical and mathematical models of the tsunami climbing a beach, Tsunami Climbing a Beach, 8-34, Applied Physics Instituts Press, Gorky, 1985. 